\title{
An evolving photoelectric efficiency at cosmic noon?
}

\author{
Jed McKinney ${ }^{1}\left(\mathbb{D}\right.$, Alexandra Pope ${ }^{1}$, Lee Armus ${ }^{2}$, Ranga Chary ${ }^{3}$, \\ Mark Dickinson ${ }^{4}$ and Allison Kirkpatrick ${ }^{5}$ \\ ${ }^{1}$ Department of Astronomy, University of Massachusetts, Amherst, MA 01003, USA \\ ${ }^{2}$ Infrared Processing and Analysis Center, MC 314-6, Caltech, 1200 E. California Blvd., \\ Pasadena, CA 91125, USA \\ ${ }^{3}$ Division of Physics, Math \& Astronomy, California Institute of Technology, Pasadena, CA \\ 91125 \\ ${ }^{4}$ National Optical Astronomy Observatory, 950 North Cherry Avenue, Tucson, AZ 85719, USA \\ ${ }^{5}$ Department of Physics \& Astronomy, University of Kansas, Lawrence, KS 66045, USA
}

\begin{abstract}
To sustain star formation rates (SFRs) of hundreds to thousands of solar masses per year over millions of years, a galaxy must efficiently cool its gas. At $z \sim 2$, the peak epoch for stellar mass assembly, tracers of gas heating and cooling remain largely unexplored. For one $z \sim 2$ starburst galaxy GS IRS20, we present Spitzer IRS spectroscopy of Polycyclic Aromatic Hydrocarbon (PAH) emission, and ALMA observations of [C II] $158 \mu \mathrm{m}$ fine-structure emission which we use to probe ISM heating/cooling. Coupled with an unusually warm dust component, the ratio of $[\mathrm{C} \mathrm{II}] / \mathrm{PAH}$ emission suggests a low photolelectric efficiency, and/or the importance of cooling from other far-IR lines in this galaxy. A low photoelectric efficiency at $z \sim 2$ could be key for the peak in the SFR density of the universe by decoupling stellar radiation from ISM gas temperatures.
\end{abstract}

Keywords. galaxies: high-redshift, galaxies: individual, galaxies: ISM, galaxies: starburst

\section{Introduction}

In galaxies, interstellar gas heats and cools during the cycle of star-formation and feedback processes. Photoelectrons ejected from polycyclic aromatic hydrocarbons (PAHs) are key for converting stellar radiation to thermal energy in photodissociation regions (PDRs), where large gas densities are illuminated by far-UV fluxes from young stars (Tielens \& Hollenbach 1985). PDRs cool predominantly via far-infrared (IR) finestructure lines such as [C II], which can contain as much as $0.1-1 \%$ of a galaxy's total IR emission ( $\left.\mathrm{L}_{\mathrm{IR}}, 8-1000 \mu \mathrm{m}\right)$. The ratio of [C II] to mid-IR PAH emission is therefore a powerful diagnostic of PDR regions of high- $z$ galaxies (Helou et al. 2001).

[C II] observations of galaxies at cosmic noon are limited to only a handful (Zanella et al. 2018), and even fewer have mid-IR PAH spectra from Spitzer (e.g., Brisbin et al. 2015). Thus, key ISM line-ratios at $z \sim 2$ have yet to be characterized. To this point, we present [C II] and PAH observations of GS IRS20, a starburst galaxy at $z=1.924$ with the highest signal-to-noise ALMA detection of [C II] at $z \sim 2$ to date (Fig. 1, Left inset). GS IRS20 has an unusually warm dust component, and is a unique opportunity to probe the ISM conditions of extreme star formation.

\section{Overview}

The luminosity ratio of $[\mathrm{C} \mathrm{II}] / \mathrm{L}_{\mathrm{IR}}$ and $\mathrm{PAH} / \mathrm{L}_{\mathrm{IR}}$ show a deficit towards higher $\mathrm{L}_{\mathrm{IR}}$, with significant scatter related to, amongst other factors, dust temperatures, geometry, 

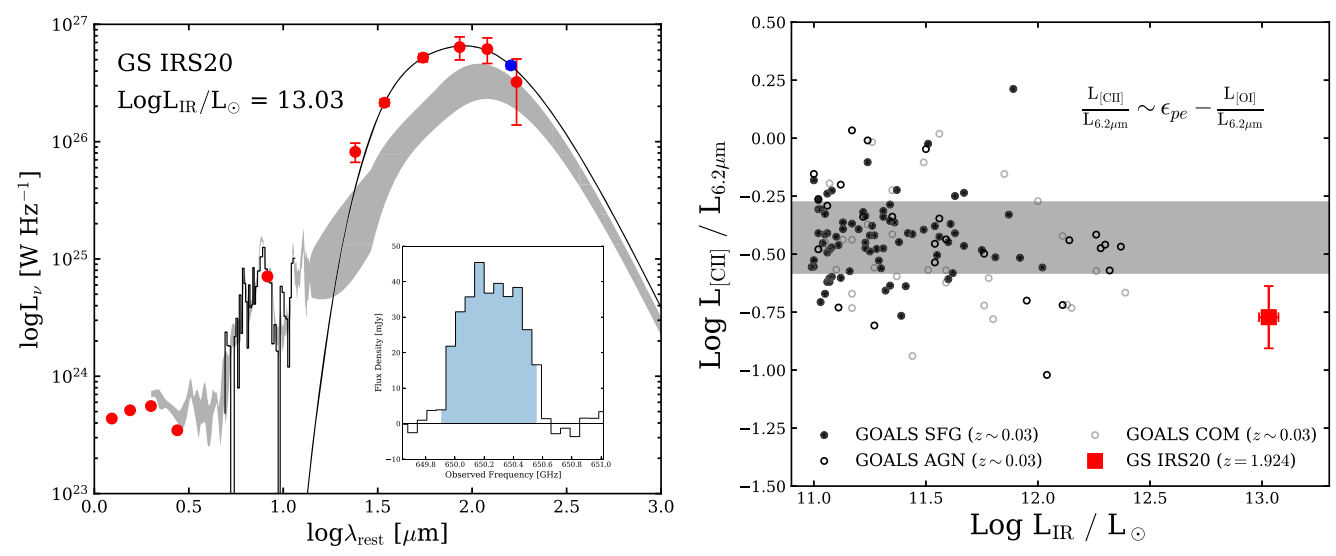

Figure 1. (Left) GS IRS20's SED with the ALMA Band 9 [C II] detection shown in the inset panel. Multi-wavelength photometry is shown with red and blue points, and its IRS spectrum is shown in black. The shaded gray region corresponds to an empirical SED from Kirkpatrick et al. (2015) normalized to GS IRS20's PAH spectrum. The thin black line is a two-temperature graybody fit to photometry past $25 \mu \mathrm{m}$. (Right) The ratio of [C II] luminosity to the $6.2 \mu \mathrm{m}$ $\mathrm{PAH}$ feature luminosity. The gray shaded region contains the $1 \sigma$ scatter about the mean of local star-forming IR galaxies from GOALS. GS IRS20 is shown in red.

and fractional contributions from different gas phases (e.g., Pope et al. 2013; Díaz-Santos et al. 2017). As noted in Helou et al. (2001), PAH emission is a more appropriate normalization factor for $\mathrm{L}_{[\mathrm{C} \text { II] }}$ as the collisional excitation of $\mathrm{C}^{+}$is proportional to the density of PAH photoelectrons. Indeed, Figure 1 (Right) shows that $\mathrm{L}_{[\mathrm{C} \mathrm{II}]} / \mathrm{L}_{6.2 \mu \mathrm{m}}$ remains relatively constant as a function of $\mathrm{L}_{\mathrm{IR}}$ in low- $z$ IR galaxies, particularly those that are purely star-forming. GS IRS20 falls below this average by $\sim 3 \sigma . \mathrm{L}_{[\mathrm{C} \text { II] }} / \mathrm{L}_{6.2 \mu \mathrm{m}}$ traces the balance of gas cooling through the $[\mathrm{C} \mathrm{II}]$ channel and photoelectric gas heating, and is therefore proportional to the difference in photoelectric efficiency $\left(\epsilon_{p e}\right)$, and cooling via $[\mathrm{O} \mathrm{I}]$ assuming other far-IR emission lines are negligible (see Eq. 7 in Croxall et al. 2012).

\section{Implications}

The photoelectric efficiency is a measure of total gas heating to total dust heating, and can fall at high ratios of far-UV flux to gas density as PAHs lose more of their surface electrons, reducing their photoelectric yields (e.g., Helou et al. 2001). As a result, PDRs become less efficient at converting stellar radiation to thermal energy in atomic gas. Empirically, Croxall et al. (2012) find lower values of $\epsilon_{p e}$ in warmer dust environments, consistent with harder and more intense far-UV radiation fields.

GS IRS20 has an unusually warm dust component compared to other $z \sim 2$ IR galaxies (Fig. 1 Left), is a starburst galaxy, and has a low $\mathrm{L}_{[\mathrm{C} \mathrm{II}]} / \mathrm{L}_{6.2 \mu \mathrm{m}}$ (Fig. 1 Right). For these reasons, $\epsilon_{p e}$ may be low in GS IRS20, and/or cooling via [O I] plays an important role. We tentatively speculate that a decrease in $\epsilon_{p e}$ in high-z star-forming galaxies could suppress stellar feedback and contribute to the peak in the IR SFR density. If this is the case, atomic gas may remain cool to form more stars, yielding higher SFRs.

\section{References}

Helou, G., Malhotra, S., Hollenbach, D. J., Dale, D. A., \& Contursi, A. 2001, ApJL, 548, L73

Tielens, A. G. G. M., \& Hollenbach, D. 1985, ApJ, 291, 722

Pope, A., Wagg, J., Frayer, D., et al. 2013, ApJ, 772, 92

Zanella, A., Daddi, E., Magdis, G., et al. 2018, MNRAS, 481, 1976 
Brisbin, D., Ferkinhoff, C., Nikola, T., et al. 2015, ApJ, 799, 13

Kirkpatrick, A., Pope, A., Sajina, A., et al. 2015, ApJ, 814, 9

Díaz-Santos, T., Armus, L., Charmandaris, V., et al. 2017, ApJ, 846, 32

Croxall, K. V., Smith, J. D., Wolfire, M. G., et al. 2012, ApJ, 747, 81 